\title{
Long-lived bino and wino in supersymmetry with heavy scalars and higgsinos
}

\author{
Krzysztof Rolbiecki $^{a}$ and Kazuki Sakurai ${ }^{b}$ \\ ${ }^{a}$ Instituto de Física Teórica, IFT-UAM/CSIC, \\ C/ Nicolás Cabrera, 13-15, Cantoblanco, 28049 Madrid, Spain \\ ${ }^{b}$ Department of Physics, King's College London, \\ London WC2R 2LS, U.K. \\ E-mail: rolbiecki.krzysztof@csic.es, kazuki.sakurai@kcl.ac.uk
}

\begin{abstract}
We point out that there is a parameter region in supersymmetry with heavy scalars and higgsinos, in which the heavier of bino and wino becomes long-lived as a consequence of the heavy higgsinos. In this region these electroweak gaugino sectors are secluded from each other with very small mixings that are inversely proportional to the higgsino mass, $\mu$. We revisit the bino and bino decays and provide simple formulae for the partial decay rates and the lifetimes in the limit of heavy higgsinos. The scale of $\mu$ required for the long-lived electroweak gauginos highly depends on the mass hierarchy between bino and wino. The neutral wino can be long-lived $(c \tau \gtrsim 1 \mathrm{~cm})$ even with $|\mu| \gtrsim 10 \mathrm{TeV}$ if $m_{\tilde{W}}-m_{\tilde{B}} \sim 20 \mathrm{GeV}$. We discuss the collider signatures of the long-lived binos and winos in this scenario.
\end{abstract}

KEYWORds: Supersymmetry Phenomenology

ArXiv EPrint: 1506.08799 


\section{Contents}

1 Introduction 1

2 Interactions between winos and bino 2

3 Two-body decays 5

3.1 Wino NLSP case $\left(\left|M_{2}\right|>\left|M_{1}\right|\right)$

3.2 Bino NLSP case $\left(\left|M_{1}\right|>\left|M_{2}\right|\right)$

4 Three-body decays $\quad 8$

4.1 Wino NLSP case $\left(\left|M_{2}\right|>\left|M_{1}\right|\right) \quad 8$

4.2 Bino NLSP case $\left(\left|M_{1}\right|>\left|M_{2}\right|\right) \quad 12$

$5 \quad$ Large mass splitting between gauginos and higgsinos 13

6 Collider signatures $\quad 14$

$\begin{array}{llr}7 & \text { Conclusions } & 16\end{array}$

$\begin{array}{ll}\text { A Auxiliary functions } & 17\end{array}$

$\begin{array}{ll}\text { B Comparison with SDecay } & 18\end{array}$

\section{Introduction}

Supersymmetry (SUSY) remains as a promising new physics candidate after LHC Run-I. The discovery of the Higgs boson with $m_{h} \simeq 125 \mathrm{GeV}[1-4]$ and the negative results of the new physics searches at the LHC make the SUSY scenario with heavy scalars more attractive. In such a scenario, scalars (except for the Standard Model (SM) like Higgs boson, $h$ ) are heavier than the $\mathrm{TeV}$ scale and the observed Higgs mass can be easily realised by the large corrections from the heavy scalars [5-7]. The gauge coupling unification can be achieved with the light gauginos [8] and such light gauginos can be within the reach of the collider experiments. If the wino is the lightest SUSY particle (LSP), the thermal relic abundance bounds the wino mass from above by $2.7-3 \mathrm{TeV}$ [9-11]. Phenomenology of light neutralinos with the thermal relic density smaller than the observed dark matter abundance was extensively studied in the literature [12-24]. The splitting between the scalar and gaugino masses is also motivated from model building perspective. If the SUSY breaking field with non-vanishing $F$-term is charged under some symmetry, only scalars acquire the soft masses at tree-level. The gaugino masses can be generated by other 
mechanisms such as the anomaly mediation, but they are parametrically smaller than the scalar masses.

It has been pointed out that gluinos can be long-lived in this scenario if squarks are heavier than $\mathcal{O}\left(10^{3}\right) \mathrm{TeV}$ [8]. Such long-lived gluinos, if produced, may leave a distinctive $R$-hadron signature or a signature of displaced vertices $[25,26]$. With decoupled squarks, the $\mathrm{SU}(3)$ gaugino sector is secluded from the other gaugino sectors and the gluino becomes stable even if there exist lighter gauginos. The situation is somewhat different for the $\mathrm{SU}(2)_{L}$ and $\mathrm{U}(1)_{Y}$ gauginos because of the electroweak (EW) symmetry breaking (EWSB). These sectors are mixed after the EWSB and the heavier gaugino can decay into lighter ones without mediation of scalars. However, this mixing is proportional to the ratios of the vacuum expectation values of the Higgs field and the mass of the fermionic component of the Higgs field: $v_{u} / \mu$ and $v_{d} / \mu$, and can become arbitrarily small if the magnitude of $\mu$ increases.

In this paper we point out that there is a region in SUSY parameter space with heavy scalars and higgsinos where the heavier of EW gauginos becomes long-lived as a consequence of the large $\mu$ parameter. We revisit the decays of EW gauginos and derive simple formulae for partial decay rates and lifetimes, which are valid in the limit of the heavy higgsinos. We identify the region where the bino and winos become long-lived for both cases of two- and three-body decays.

The paper is organised as follows. In the following section we review the chargino and neutralino sectors of the minimal supersymmetric Standard Model (MSSM) and study the mixings and interactions between bino and winos in the heavy higgsino limit. We then revisit the two- and three-body decays of bino and winos in sections 3 and 4, respectively. In these sections, we identify the scale of $\mu$, at which the bino and winos become longlived in collider experiments. In section 5 we briefly discuss how the large mass splitting between the gauginos and higgsinos can be theoretically achieved. The collider signatures of the long-lived bino and winos in our scenario is briefly discussed in section 6. Finally, we conclude in section 7 . In appendix A we list detailed formulae for auxiliary functions used in the paper and in appendix B we compare approximate expressions obtained here with an exact calculation using SDecay [27].

\section{Interactions between winos and bino}

The chargino mass matrix is given by: ${ }^{1}$

$$
\mathcal{M}_{C}=\left(\begin{array}{cc}
M_{2} & \sqrt{2} m_{W} s_{\beta} \\
\sqrt{2} m_{W} c_{\beta} & \mu
\end{array}\right),
$$

where $c_{\beta}\left(s_{\beta}\right)$ represents $\cos \beta(\sin \beta)$ and $\beta$ is defined as the ratio of the vacuum expectation values of two Higgs fields, $\tan \beta=\left\langle H_{u}\right\rangle /\left\langle H_{d}\right\rangle$. In the large $\mu$ limit, this matrix can be

\footnotetext{
${ }^{1}$ We closely follow the calculation and conventions used in [28, 29].
} 
diagonalised as $\mathcal{M}_{C}^{\text {diag }}=U^{*} \mathcal{M}_{C} V^{-1}$ where $U$ and $V$ take the following forms

$$
U \simeq\left(\begin{array}{cc}
1 & -\frac{\sqrt{2} s_{\beta} m_{W}}{\mu} \\
\frac{\sqrt{2} s_{\beta} m_{W}}{\mu} & 1
\end{array}\right), \quad V \simeq\left(\begin{array}{cc}
1 & -\frac{\sqrt{2} c_{\beta} m_{W}}{\mu} \\
\frac{\sqrt{2} c_{\beta} m_{W}}{\mu} & 1
\end{array}\right) .
$$

The eigenvalues are then given by:

$$
m_{\tilde{W}^{ \pm}} \simeq M_{2}, \quad m_{\tilde{\chi}_{2}^{ \pm}} \simeq|\mu|,
$$

with $m_{\tilde{W}^{ \pm}} \ll m_{\tilde{\chi}_{2}^{ \pm}} \cdot{ }^{2}$

The neutralino mass matrix is given by

$$
\mathcal{M}_{N}=\left(\begin{array}{cccc}
M_{1} & 0 & -m_{Z} s_{W} c_{\beta} & m_{Z} s_{W} s_{\beta} \\
0 & M_{2} & m_{Z} c_{W} c_{\beta} & -m_{Z} c_{W} s_{\beta} \\
-m_{Z} s_{W} c_{\beta} & m_{Z} c_{W} c_{\beta} & 0 & -\mu \\
m_{Z} s_{W} s_{\beta} & -m_{Z} c_{W} s_{\beta} & -\mu & 0
\end{array}\right) .
$$

In the large $\mu$ limit, the diagonalising matrix $Z$ can be written as

$$
Z \simeq\left(\begin{array}{cccc}
1 & -\frac{m_{Z}^{2} s_{2 W} s_{2 \beta}}{2 \mu\left(M_{2}-M_{1}\right)} & \frac{m_{Z} s_{W} s_{\beta}}{\mu} & -\frac{m_{Z} s_{W} c_{\beta}}{\mu} \\
\frac{m_{Z}^{2} s_{2} s_{2 \beta}}{2 \mu\left(M_{2}-M_{1}\right)} & 1 & -\frac{m_{Z} c_{W} s_{\beta}}{\mu} & \frac{m_{Z} c_{W} c_{\beta}}{\mu} \\
\frac{m_{Z} s_{W}\left(c_{\beta}-s_{\beta}\right)}{\sqrt{2} \mu} & -\frac{m_{Z} c_{W}\left(c_{\beta}-s_{\beta}\right)}{\sqrt{2} \mu} & \frac{1}{\sqrt{2}} & \frac{1}{\sqrt{2}} \\
\frac{m_{Z} s_{W}\left(c_{\beta}+s_{\beta}\right)}{\sqrt{2} \mu} & -\frac{m_{Z} c_{W}\left(c_{\beta}+s_{\beta}\right)}{\sqrt{2} \mu} & -\frac{1}{\sqrt{2}} & \frac{1}{\sqrt{2}}
\end{array}\right),
$$

where $s_{W}$ is the sine of Weinberg angle, $\sin \theta_{W}$, and $c_{W} \equiv \cos \theta_{W}$. The mass eigenvalues are found to be

$$
m_{\tilde{B}} \simeq M_{1}, \quad m_{\tilde{W}^{0}} \simeq M_{2}, \quad m_{\tilde{\chi}_{3}^{0}} \simeq m_{\tilde{\chi}_{4}^{0}} \simeq|\mu|,
$$

where $m_{\tilde{B}}, m_{\tilde{W}^{0}} \ll m_{\tilde{\chi}_{3}^{0}}, m_{\tilde{\chi}_{4}^{0}}$.

The wino and bino interact with each other and a Higgs or a gauge boson through the mixing matrices $U, V$ and $Z$. The interaction with the light Higgs boson, $h$, is dictated by the $\tilde{\chi}_{j}^{0}-\tilde{\chi}_{i}^{0}$ - $h$ coupling [29]

$$
G_{i j h}^{L}=G_{i j h}^{R}=-\frac{1}{2 s_{W}}\left(Z_{j 2}-Z_{j 1} \tan \theta_{W}\right)\left(Z_{i 3} \sin \alpha+Z_{i 4} \cos \alpha\right)+i \leftrightarrow j,
$$

where $\alpha$ is the mixing angle of the neutral Higgs sector and reduces to $\sin \alpha=c_{\beta}, \cos \alpha=$ $-s_{\beta}$ in the decoupling limit of the SUSY Higgses [30-32]. In the large $\mu$ limit these couplings can be written as

$$
G_{\tilde{W}^{0} \tilde{B} h} \simeq \frac{1}{2 s_{W}}\left[\left(c_{\beta} Z_{13}-s_{\beta} Z_{14}\right)-\frac{s_{W}}{c_{W}}\left(c_{\beta} Z_{23}-s_{\beta} Z_{24}\right)\right] \simeq \frac{m_{Z} s_{2 \beta}}{\mu} .
$$

This interaction is illustrated diagrammatically in the top-row graphs of figure 1 . We note that the coupling originates from the bino-higgsino and wino-higgsino mixing and is suppressed by $m_{Z} / \mu$ for large $\mu$.

\footnotetext{
${ }^{2}$ In order to stress wino nature of the lightest chargino, we denote it here as $\tilde{W}^{ \pm}$rather then usual $\tilde{\chi}_{1}^{ \pm}$.
} 

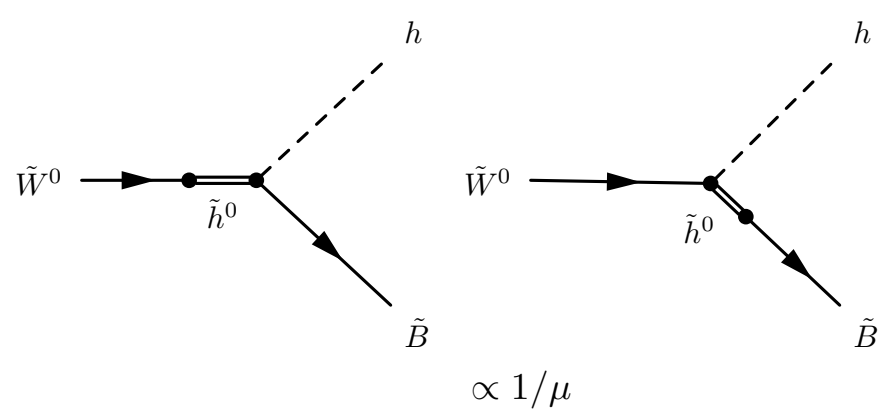

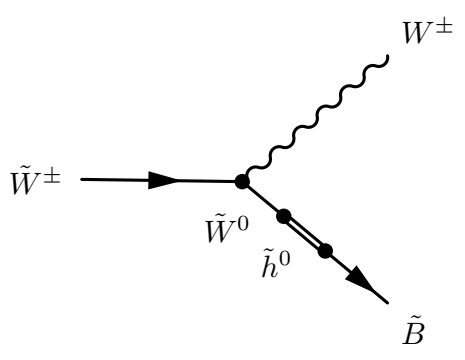

$\propto 1 / \mu$

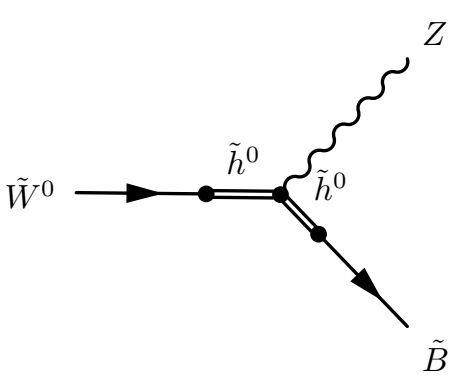

$\propto 1 / \mu^{2}$

Figure 1. The wino-bino interaction with the SM Higgs and gauge bosons.

The interaction with the $W$ boson is described by the $\tilde{\chi}_{j}^{ \pm}-\tilde{\chi}_{i}^{0}-W^{\mp}$ coupling

$$
G_{i j W}^{L}=\frac{1}{s_{W}}\left(Z_{i 2} V_{j 1}-\frac{1}{\sqrt{2}} Z_{i 4} V_{j 2}\right), \quad G_{i j W}^{R}=\frac{1}{s_{W}}\left(Z_{i 2} U_{j 1}+\frac{1}{\sqrt{2}} Z_{i 3} U_{j 2}\right),
$$

and in the large $\mu$ limit these couplings can be written as

$$
G_{\tilde{B} \tilde{W} \pm W} \simeq-\frac{m_{Z}^{2} c_{W} s_{2 \beta}}{\mu\left(M_{2}-M_{1}\right)} .
$$

The interaction is depicted in the bottom-left graph of figure 1. The coupling is proportional to $m_{Z} / \mu$ and originates from the bino-wino mixing, $Z_{12}$.

We illustrate the wino-bino interaction with the $Z$ boson in the bottom-right graph of figure 1. Unlike the other interactions, this coupling requires both the wino-higgsino and the bino-higgsino interaction. Formally, the interaction is defined by the $\tilde{\chi}_{j}^{0}-\tilde{\chi}_{i}^{0}-Z$ coupling

$$
G_{i j Z}^{R}=-G_{i j Z}^{L}=\frac{1}{s_{2 W}}\left(Z_{i 3} Z_{j 3}-Z_{i 4} Z_{j 4}\right),
$$

and reduces in the large $\mu$ limit to

$$
G_{\tilde{W}^{0} \tilde{B} Z}^{R}=-G_{\tilde{W}^{0} \tilde{B} Z}^{L} \simeq \frac{m_{Z}^{2} c_{2 \beta}}{2 \mu^{2}}
$$

These couplings are proportional to $\left(m_{Z} / \mu\right)^{2}$ and become small quicker than the other couplings as $|\mu|$ increases. There exists another contribution to the bino-wino- $Z$ interaction from higher order terms. The higgsino-Higgs loop diagram generates a dimension-5 operator,

$$
\tilde{B} \tilde{W}^{a} \sigma^{\mu \nu} F_{\mu \nu}^{a}
$$


A naive dimensional analysis suggests that the coefficient of this operator is proportional to $\alpha /(4 \pi \mu)$. Although this contribution is suppressed by the loop factor, it can easily dominate the tree-level interaction, eq. (2.12), for very large $\mu$. We leave a detailed study of effects of this operator for future work.

\section{Two-body decays}

\subsection{Wino NLSP case $\left(\left|M_{2}\right|>\left|M_{1}\right|\right)$}

Throughout this paper, we assume that gluinos are heavier than wino and bino. In this section we consider the cases where winos are heavier than bino. In SUSY models with heavy scalars and higgsinos, the mass difference between the charged and neutral winos is small and the decays among the wino multiplet (e.g. $\tilde{W}^{ \pm} \rightarrow \pi^{ \pm} \tilde{W}^{0}$ ) can be neglected compared to the wino decays into a bino. We therefore do not consider the decays within the wino multiplet in this section. We will mention the effect of this decay mode in section 6 .

The decay mode of the charged wino is shown in the bottom-left diagram of figure 1 . The final state consists of $W^{ \pm}$and $\tilde{B}$. The decay rate of this process is given by [29]:

$$
\begin{aligned}
\Gamma\left(\tilde{W}^{ \pm} \rightarrow W^{ \pm} \tilde{B}\right) & =\frac{\alpha}{4} M_{2} G_{\tilde{B} \tilde{W}^{ \pm} W}^{2} f_{-}\left(\mu_{\tilde{B}}, \mu_{W}\right) \\
& \stackrel{\mu}{\simeq} \frac{\alpha s_{2 \beta} M_{2}}{4} \frac{m_{Z}^{2}}{\mu^{2}} \frac{m_{W}^{2} f_{-}\left(\mu_{\tilde{B}}, \mu_{W}\right)}{\left(M_{2}-M_{1}\right)^{2}} \\
M_{2} \gg m_{W} & \frac{\alpha}{4}\left(M_{2}-M_{1}\right) s_{2 \beta}^{2} \frac{m_{Z}^{2}}{\mu^{2}}\left(1+\frac{M_{1}}{M_{2}}\right)^{3},
\end{aligned}
$$

where $\mu_{\tilde{B}}=M_{1}^{2} / M_{2}^{2}$ and $\mu_{W}=m_{W}^{2} / M_{2}^{2}$. The analytic expression for $f_{-}(x, y)$ is given in appendix A. Throughout this and the following sections, the first approximation is valid in the large $\mu$ limit, while the following assumes both $\mu \gg m_{Z}$ and $M_{2} \gg m_{W}$. Using the last expression, the lifetime of the charged wino is found to be

$$
c \tau_{\tilde{W}^{ \pm}} \simeq 2.5 \mathrm{~cm} \cdot\left(\frac{\mu}{10^{6} \mathrm{TeV}}\right)^{2}\left(\frac{500 \mathrm{GeV}}{M_{2}-M_{1}}\right)\left(\frac{1}{s_{2 \beta}}\right)^{2}\left(\frac{M_{2}}{M_{2}+M_{1}}\right)^{3} .
$$

In figure 2(a) we show the decay rate of $\tilde{W}^{ \pm}$as a function of $|\mu|$. The horizontal green line denotes the decay rate of $B^{ \pm}$meson as a reference, where the displacement of the $\tilde{W}^{ \pm}$ decay from the primary vertex starts to be visible. The horizontal blue line corresponds to the decay rate where the $c / \Gamma=1 \mathrm{~cm}$, where the charged winos start to reach the trackers leaving the distinctive kink-like signature. ${ }^{3}$ As can be seen, the charged wino has a collider relevant lifetime for $|\mu| \gtrsim \mathcal{O}\left(10^{5}\right) \mathrm{TeV}$, if the two body $\tilde{W}^{ \pm} \rightarrow W^{ \pm} \tilde{B}$ is kinematically allowed.

There are two possible decay modes for the neutral wino: $\tilde{W}^{0} \rightarrow Z \tilde{B}$ and $\tilde{W}^{0} \rightarrow h \tilde{B}$. The former is depicted in the bottom-right diagram in figure 1 and can be mediated either by the coupling eq. (2.11) or by the dimension-5 operator, eq. (2.13). However, these

\footnotetext{
${ }^{3}$ The trackers inner radii are for example: $44 \mathrm{~mm}$ for CMS [33], $50.5 \mathrm{~mm}$ for ATLAS [34], and $16 \mathrm{~mm}$ for ILD at the planned International Linear Collider [35].
} 


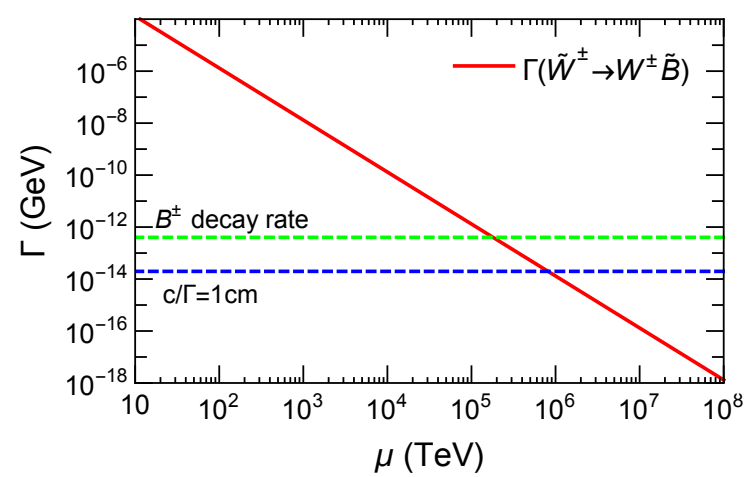

(a)

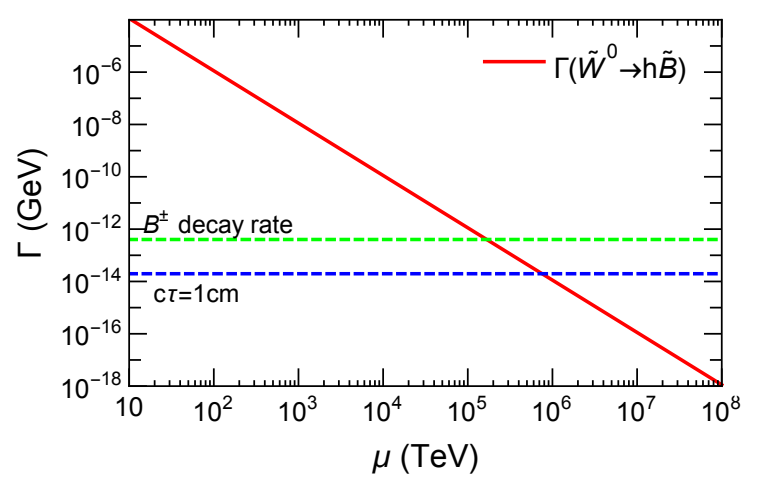

(b)

Figure 2. The two-body decay rate of winos as a function of $\mu$, with other parameters fixed at: $M_{2}=800 \mathrm{GeV}, M_{1}=300 \mathrm{GeV}, \tan \beta=2$.

contributions are suppressed by the extra $m_{Z} / \mu$ factor and the loop factor in the matrix element, respectively, compared to the $\tilde{W}^{0} \rightarrow h \tilde{B}$ decay. Therefore, if the mass difference is large enough to allow the two body $\tilde{W}^{0} \rightarrow h \tilde{B}$ decay, the neutral wino predominantly decays into $h$ and $\tilde{B}$. The decay rate is given by:

$$
\begin{aligned}
\Gamma\left(\tilde{W}^{0} \rightarrow h \tilde{B}\right) & =\frac{\alpha}{4} M_{2} G_{\tilde{W}^{0} \tilde{B} h}^{2} f_{h}\left(\mu_{\tilde{B}}, \mu_{h}\right) \\
& \stackrel{\Perp}{\simeq} \frac{\alpha M_{2}}{4} s_{2 \beta}^{2} \frac{m_{Z}^{2}}{\mu^{2}} f_{h}\left(\mu_{\tilde{B}}, \mu_{h}\right) \\
& \stackrel{\simeq}{M_{2} \gg m_{h}} \frac{\alpha}{4}\left(M_{2}-M_{1}\right) s_{2 \beta}^{2} \frac{m_{Z}^{2}}{\mu^{2}}\left(1+\frac{M_{1}}{M_{2}}\right)^{3},
\end{aligned}
$$

where $\mu_{h}=m_{h}^{2} / M_{2}^{2}$, and the analytic expression of $f_{h}(x, y)$ is given in appendix A. We approximate the exact formula for $\mu \gg m_{Z}$ and, additionally, $M_{2} \gg m_{h}$ in the second and third line, respectively. It is worth noting that the last expression is identical to the one in eq. (3.1). This can be also seen in figure 2(b), where we show the decay rate of $\tilde{W}^{0}$ as a function of $|\mu|$. The approximate lifetime formula for the $\tilde{W}^{0}$ is therefore the same as in eq. (3.2) with $c \tau_{\tilde{W}^{0}} \sim c \tau_{\tilde{W}^{ \pm}}$. At colliders, the long-lived neutral wino leaves the displaced $\operatorname{dijet/jets~signatures~[36,~37].~}$

Finally, we would like to comment on a special case when $m_{Z}<\left|M_{2}\right|-\left|M_{1}\right|<m_{h}$. In this case the $\tilde{W}^{0} \rightarrow h \tilde{B}$ decay is kinematically forbidden and the $\tilde{W}^{0}$ decays predominantly into $Z$ and $\tilde{B}$, through the bottom-right diagram in figure 1 and the dimension-5 operator, eq. (2.13). Since the tree-level contribution is suppressed by the extra $1 / \mu$ factor, the contribution from the dimension-5 operator dominates in the large $|\mu|$ region. This parameter region is interesting, because the neutral wino can be long lived with much smaller $|\mu|$. We leave this question for future work.

\subsection{Bino NLSP case $\left(\left|M_{1}\right|>\left|M_{2}\right|\right)$}

If bino is heavier than winos, the bino can decay either to $\tilde{B} \rightarrow \tilde{W}^{ \pm} W^{\mp}$, or $\tilde{B} \rightarrow h \tilde{W}^{0}$, or $\tilde{B} \rightarrow Z \tilde{W}^{0}$. As we discussed in the previous subsection, the interaction $\tilde{B}-\tilde{W}-Z$ is 


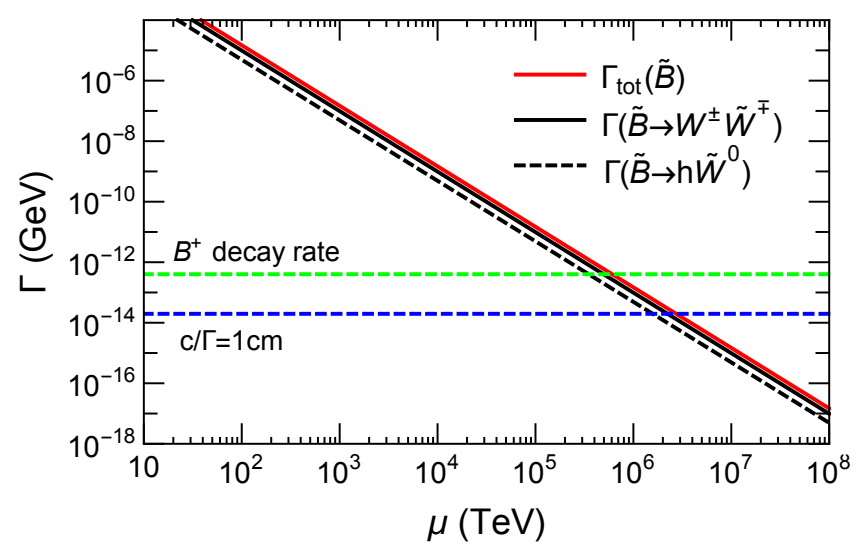

Figure 3. The two-body decay rate of bino as a function of $\mu$, with other parameters fixed at: $M_{1}=800 \mathrm{GeV}, M_{2}=300 \mathrm{GeV}, \tan \beta=2$.

suppressed compared to $\tilde{B}-\tilde{W}-W$ and $\tilde{B}-\tilde{W}-h$, and the bino decay is dominated by the $W$ and $h$ decay modes. The partial decay rates for these modes are given by:

$$
\begin{aligned}
& \Gamma\left(\tilde{B} \rightarrow \tilde{W}^{ \pm} W^{\mp}\right) \quad=\quad \frac{\alpha}{2} M_{1} G_{\tilde{B} \tilde{W}^{ \pm} W}^{2} f_{-}\left(\mu_{\tilde{W}}, \mu_{W}\right) \\
& \stackrel{\mu \gg m_{Z}}{\simeq} \frac{\alpha s_{2 \beta}^{2} M_{1}}{2} \frac{m_{Z}^{2}}{\mu^{2}} \frac{m_{W}^{2} f_{-}\left(\mu_{\tilde{W}}, \mu_{W}\right)}{\left(M_{1}-M_{2}\right)^{2}} \\
& \stackrel{M_{1} \gg m_{W}}{\simeq} \frac{\alpha}{2}\left(M_{1}-M_{2}\right) s_{2 \beta}^{2} \frac{m_{Z}^{2}}{\mu^{2}}\left(1+\frac{M_{2}}{M_{1}}\right)^{3},
\end{aligned}
$$

and

$$
\begin{aligned}
\Gamma\left(\tilde{B} \rightarrow h \tilde{W}^{0}\right) & =\frac{\alpha}{4} M_{1} G_{\tilde{W}^{0} \tilde{B} h}^{2} f_{h}\left(\mu_{\tilde{W}}, \mu_{h}\right) \\
& \stackrel{\simeq}{\simeq} \frac{\alpha M_{1}}{4} s_{2 \beta}^{2} \frac{m_{Z}^{2}}{\mu^{2}} f_{h}\left(\mu_{\tilde{W}}, \mu_{h}\right) \\
& \stackrel{\simeq}{M_{1}} m_{h} \frac{\alpha}{4}\left(M_{1}-M_{2}\right) s_{2 \beta}^{2} \frac{m_{Z}^{2}}{\mu^{2}}\left(1+\frac{M_{2}}{M_{1}}\right)^{3},
\end{aligned}
$$

where $\mu_{\tilde{W}}=M_{2}^{2} / M_{1}^{2}, \mu_{W}=m_{W}^{2} / M_{1}^{2}$ and $\mu_{h}=m_{h}^{2} / M_{1}^{2}$. The approximate formulae are for successively applied $M_{1} \gg m_{W}$ and $M_{1} \gg m_{h}$, respectively. If $M_{1} \gg m_{h}$, we find that the branching ratios for different decay modes are approximately equal:

$$
\operatorname{BR}\left(\tilde{B} \rightarrow W^{+} \tilde{W}^{-}\right)=\operatorname{BR}\left(\tilde{B} \rightarrow W^{-} \tilde{W}^{+}\right) \sim \mathrm{BR}\left(\tilde{B} \rightarrow h \tilde{W}^{0}\right) \sim 1 / 3 .
$$

Figure 3 shows the total and partial decay rates for the bino as a function of $|\mu|$. One can see that the bino can be long-lived on collider time-scales if $|\mu| \gtrsim \mathcal{O}\left(10^{6}\right) \mathrm{TeV}$. The bino lifetime is approximately given as

$$
c \tau_{\tilde{B}} \simeq 1 \mathrm{~cm} \cdot\left(\frac{\mu}{10^{6} \mathrm{TeV}}\right)^{2}\left(\frac{500 \mathrm{GeV}}{M_{1}-M_{2}}\right)\left(\frac{1}{s_{2 \beta}}\right)^{2}\left(\frac{M_{1}}{M_{1}+M_{2}}\right)^{3} .
$$




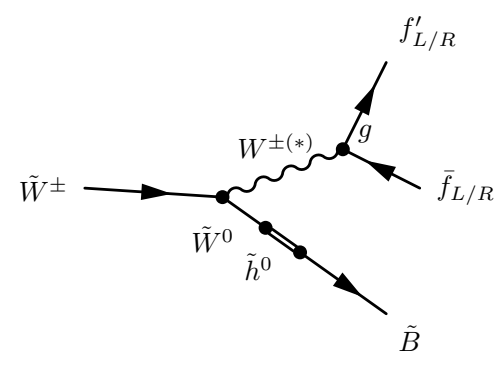

$\propto g / \mu$

Figure 4. Diagram for $\tilde{W}^{ \pm} \rightarrow f^{\prime} \bar{f} \tilde{B}$ three-body decay.

\section{Three-body decays}

In this section we consider the cases where the mass difference between bino and wino is small and two-body decay modes considered in the previous section are kinematically not allowed.

\subsection{Wino NLSP case $\left(\left|M_{2}\right|>\left|M_{1}\right|\right)$}

We start with a case of $\left|M_{2}\right|>\left|M_{1}\right|$ and define the mass difference $\Delta M \equiv\left|M_{2}\right|-\left|M_{1}\right|>0$. If $\Delta M<m_{W}$ the charged wino two-body decay, $\tilde{W}^{ \pm} \rightarrow W^{ \pm} \tilde{B}$, is forbidden. In this case, the charged wino decays into a pair of fermions and a bino, via an off-shell $W$ as shown in figure 4 . The decay rate of this process is given by:

$$
\begin{aligned}
\Gamma_{f}\left(\tilde{W}^{+} \rightarrow f \bar{f}^{\prime} \tilde{B}\right) & =\frac{\alpha^{2} M_{2}}{16 \pi} \frac{1}{s_{W}^{2}} \cdot G_{\tilde{B} \tilde{W}^{ \pm} W}^{2} \cdot \Omega_{-}\left(\mu_{\tilde{B}}, \mu_{W}\right) \\
& \stackrel{\cong}{\simeq} \frac{\alpha^{2} M_{2}}{16 \pi} \frac{s_{2 \beta}^{2}}{s_{W}^{2}} \frac{m_{Z}^{2}}{\mu^{2}} \frac{m_{W}^{2} \Omega_{-}\left(\mu_{\tilde{B}}, \mu_{W}\right)}{\left(M_{2}-M_{1}\right)^{2}} \\
\Delta & \stackrel{\geqq}{\simeq M_{2}} \frac{4 \alpha^{2}}{15 \pi} \frac{(\Delta M)^{3}}{\mu^{2}} \frac{s_{2 \beta}^{2}}{s_{2 W}^{2}},
\end{aligned}
$$

where $\mu_{W}=m_{W}^{2} / M_{2}^{2}$ and an analytic expression of $\Omega_{ \pm}(x, y)$ is given in appendix A, eqs. (A.5)-(A.9). The last approximation assumes $\Delta M \ll M_{2}$ together with $\mu \gg m_{Z}$. One may naively expect that the decay rate should be proportional to $(\Delta M)^{5} / m_{W}^{4}$ due to the $s$-channel structure. However the wino-bino mixing, $Z_{12}$, is inversely proportional to $\Delta M$, see eq. (2.5), and the decay rate is finally proportional to $(\Delta M)^{3}$.

We show the $\tilde{W}^{ \pm}$decay rate, $\Gamma\left(\tilde{W}^{ \pm} \rightarrow f \bar{f}^{\prime} \tilde{B}\right)=\sum_{f} \Gamma_{f}\left(\tilde{W}^{ \pm} \rightarrow f \bar{f}^{\prime} \tilde{B}\right)$, in figure $5($ a) and 5 (b) for $\Delta M=50$ and $20 \mathrm{GeV}$, respectively. It is clear that the charged wino can be long lived for $|\mu| \gtrsim \mathcal{O}\left(10^{3-4}\right) \mathrm{TeV}$ depending on the mass splitting $\Delta M$. The approximate formula for the charged wino lifetime is given by:

$$
c \tau_{\tilde{W}^{ \pm}} \simeq 1 \mathrm{~cm} \cdot\left(\frac{\mu}{10^{4} \mathrm{TeV}}\right)^{2}\left(\frac{30 \mathrm{GeV}}{\Delta M}\right)^{3}\left(\frac{1}{s_{2 \beta}}\right)^{2} .
$$

We now turn to the three-body decay of the neutral wino for $\Delta M<m_{Z}$. In the previous section we have noted that the $\tilde{W}-\tilde{B}-Z$ coupling is suppressed by an additional 


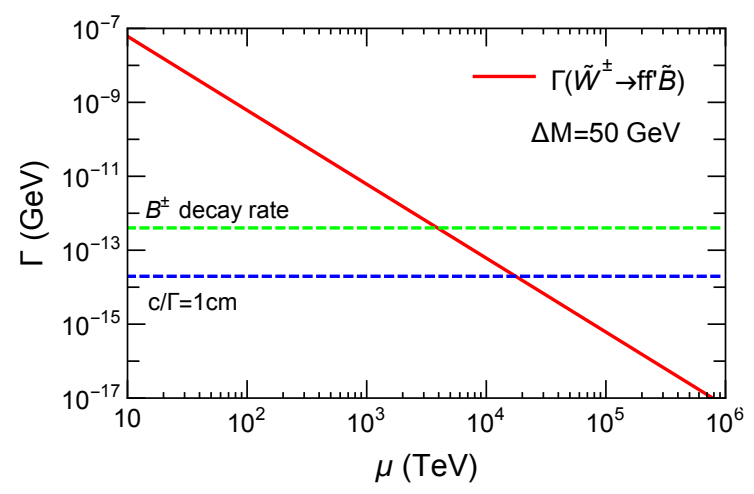

(a)

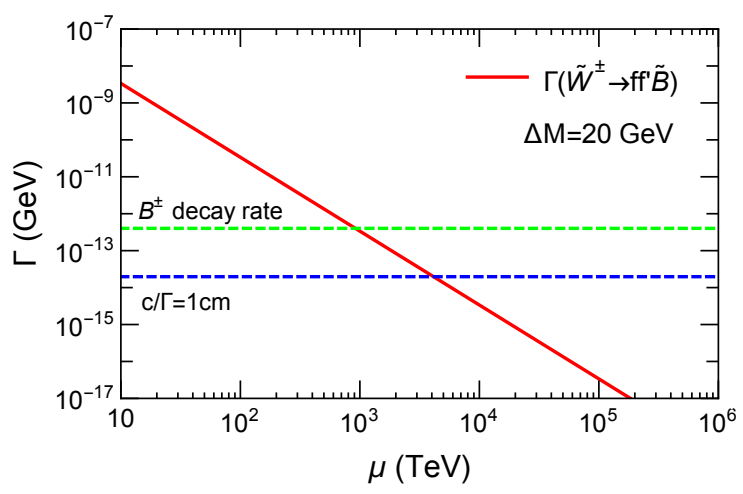

(b)

Figure 5. The three-body decay rate of the charged wino as a function of $|\mu|$ for two values of $\Delta M=50 \mathrm{GeV}$ and $20 \mathrm{GeV}$, left and right panel, respectively. The other parameters are fixed at: $M_{2}=M_{1}+\Delta M, M_{1}=500 \mathrm{GeV}, \tan \beta=2$.

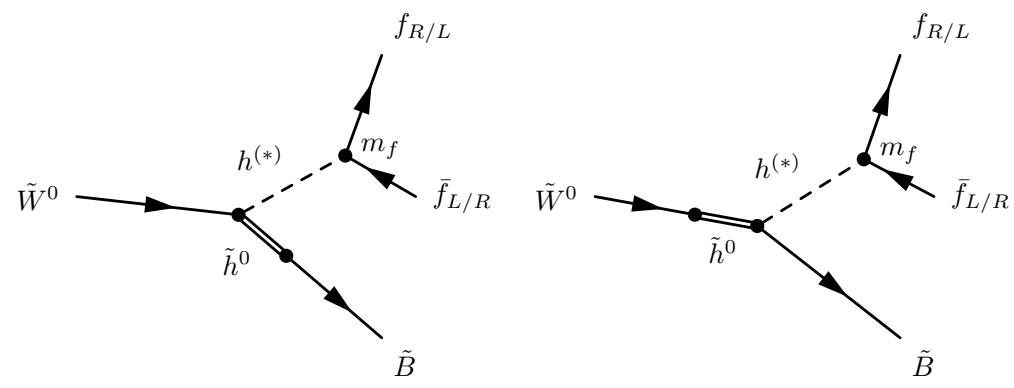

$$
\mathcal{M}_{h}^{L R} \propto m_{f} / \mu
$$

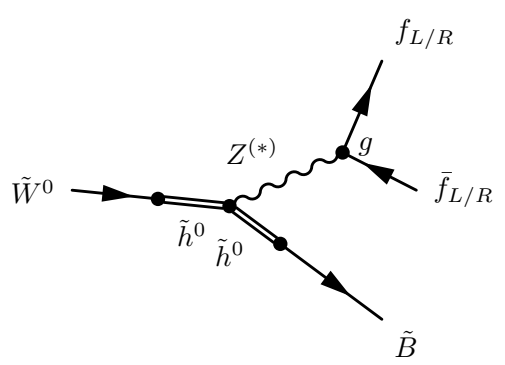

$\mathcal{M}_{Z}^{L L / R R} \propto g / \mu^{2}$

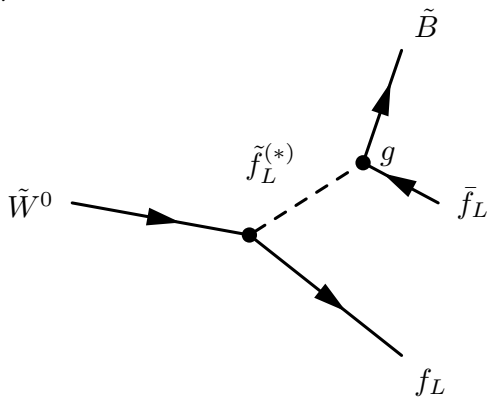

$\mathcal{M}_{\tilde{f}}^{L L} \propto g / m_{\tilde{f}_{L}}^{2}$

Figure 6. Diagrams contributing to $\tilde{W}^{0} \rightarrow f \bar{f} \tilde{B}$ three-body decay.

$m_{Z} / \mu$ factor compared to the $\tilde{W}-\tilde{B}$ - $h$ coupling, and therefore the decay mode to the Higgs dominates in the neutral wino two-body decay. In three-body decay, however, the Higgs exchange diagram also receives a suppression, which is proportional to the mass of the final state fermions as shown in the upper diagrams in figure 6.

There are several competing contributions depending on the value of $|\mu|$. Firstly, there is a $Z$-exchange diagram shown in the lower-left panel of figure 6 . The matrix element of this diagram has the $L L$ and $R R$ chirality structure for the final state fermions and is 
proportional to $1 / \mu^{2}$. Additionally, if sfermions masses are of the same order as $|\mu|$, the sfermion exchange diagram shown in the lower-right panel of figure 6 can provide a sizeable contribution. The matrix element is inversely proportional to the squared mass of the lefthanded sfermions, $m_{\tilde{f}_{L}}^{2}$. The contribution can interfere with the $Z$-exchange diagram since the matrix element has the $L L$ structure. These contributions become significant if $|\mu|$ or $m_{\tilde{f}_{L}}^{2}$ are relatively small.

Let us assume that sfermions and higgsinos have the same mass scale, $M_{X}$, which is much larger than $m_{Z}$. Neglecting the contribution induced from the dimension-5 operator, the total decay rate can be written as

$$
\Gamma\left(\tilde{W}^{0} \rightarrow f \bar{f} \tilde{B}\right)=\Gamma^{(2)}\left(\tilde{W}^{0} \rightarrow f \bar{f} \tilde{B}\right)+\Gamma^{(4)}\left(\tilde{W}^{0} \rightarrow f \bar{f} \tilde{B}\right),
$$

where $\Gamma^{(2)}=\sum_{f} \hat{\Gamma}_{f}^{h}$ is the Higgs exchange contribution which scales as $1 / M_{X}^{2}$, whilst $\Gamma^{(4)}=\sum_{f}\left(\hat{\Gamma}_{f}^{Z}+\hat{\Gamma}_{f}^{\tilde{f}}+\hat{\Gamma}_{f}^{V \tilde{f}}\right)$ is the contributions from the $Z$-exchange, sfermion exchange and their interference, scaling as $1 / M_{X}^{4} \cdot{ }^{4}$

The analytic expression for the Higgs exchange contribution, $\hat{\Gamma}_{f}^{h}$, is given by:

$$
\begin{aligned}
\hat{\Gamma}_{f}^{h}\left(\tilde{W}^{0} \rightarrow f \bar{f} \tilde{B}\right) & =\frac{\alpha^{2} M_{2}}{32 \pi} \frac{1}{s_{W}^{2}} \cdot\left(\frac{m_{f}}{m_{W}} r_{2}^{f}\right)^{2} \cdot G_{\tilde{W}^{0} \tilde{B} h}^{2} \cdot \Omega_{h}\left(\mu_{\tilde{B}}, \mu_{h}\right) \\
\mu, m_{A} \gg m_{Z} & \frac{\alpha^{2} M_{2}}{8 \pi} \frac{s_{2 \beta}^{2}}{s_{2 W}^{2}} \frac{m_{f}^{2}}{\mu^{2}} \Omega_{h}\left(\mu_{\tilde{B}}, \mu_{h}\right) \\
\Delta M & \cong M_{2} \\
& \frac{4 \alpha^{2}}{15 \pi} \frac{(\Delta M)^{5}}{m_{h}^{4}} \frac{s_{2 \beta}^{2}}{s_{2 W}^{2}} \frac{m_{f}^{2}}{\mu^{2}},
\end{aligned}
$$

where $\mu_{h}=m_{h}^{2} / M_{2}^{2}$ and $r_{2}^{u}=c_{\alpha} / s_{\beta}$ and $r_{2}^{d}=-s_{\alpha} / c_{\beta}$, both of which are reduced to -1 in the decoupling limit of the SUSY Higgs bosons $\left(m_{A} \gg m_{Z}\right)$. An analytic form of $\Omega_{h}(x, y)$ is given in appendix A, eqs. (A.10)-(A.11). In the last step we approximate the expression assuming $\Delta M \ll M_{2}$.

In the limit of heavy sfermions and higgsinos, $\hat{\Gamma}_{f}^{Z}, \hat{\Gamma}_{f}^{\tilde{f}}$ and $\hat{\Gamma}_{f}^{V \tilde{f}}$ are given by

$$
\begin{aligned}
& \hat{\Gamma}_{f}^{Z}\left(\tilde{W}^{0} \rightarrow f \bar{f} \tilde{B}\right) \quad \stackrel{\mu \gg m_{Z}}{\simeq} \quad \frac{\alpha^{2} M_{2}}{32 \pi} \frac{\kappa_{Z}^{f} c_{2 \beta}^{2}}{2 s_{2 W}^{2}} \frac{m_{Z}^{4}}{\mu^{4}} \Omega_{+}\left(\mu_{\tilde{B}}, \mu_{Z}\right) \\
& \Delta M \underset{\simeq}{\simeq} \frac{\alpha^{2}}{20 \pi} \kappa_{Z}^{f} \frac{c_{2 \beta}^{2}}{s_{2 W}^{2}} \frac{(\Delta M)^{5}}{\mu^{4}}, \\
& \hat{\Gamma}_{f}^{\tilde{f}}\left(\tilde{W}^{0} \rightarrow f \bar{f} \tilde{B}\right) \stackrel{\mu, m_{\tilde{f}_{L}} \gg m_{Z}}{\simeq} \frac{\alpha^{2} M_{2}}{32 \pi} \hat{\kappa}_{f}^{2} \frac{M_{2}^{4}}{m_{\tilde{f}_{L}}^{4}} \Omega_{f}\left(\mu_{\tilde{B}}\right) \\
& \Delta M \lll M_{2} \quad \frac{\alpha^{2}}{12 \pi} \hat{\kappa}_{f}^{2} \frac{(\Delta M)^{5}}{m_{\tilde{f}_{L}}^{4}},
\end{aligned}
$$

\footnotetext{
${ }^{4}$ The $Z$-exchange contribution induced from the dimension-5 operator has scaling $1 / M_{X}$ in the matrix element and interferes with the tree-level $Z$ and sfermion contributions. We neglect this contribution in this study because the operator should be suppressed by the loop factor $\mathcal{O}(\alpha / 4 \pi)$, which is seemingly smaller than the $m_{f} / m_{W}$ factor for $f=b, \tau$ in the tree-level Higgs exchange contribution. The full calculation including the higher order $Z$-exchange contribution is however important to precisely determine the lifetime of the neutral wino, which is beyond the scope of this paper.
} 


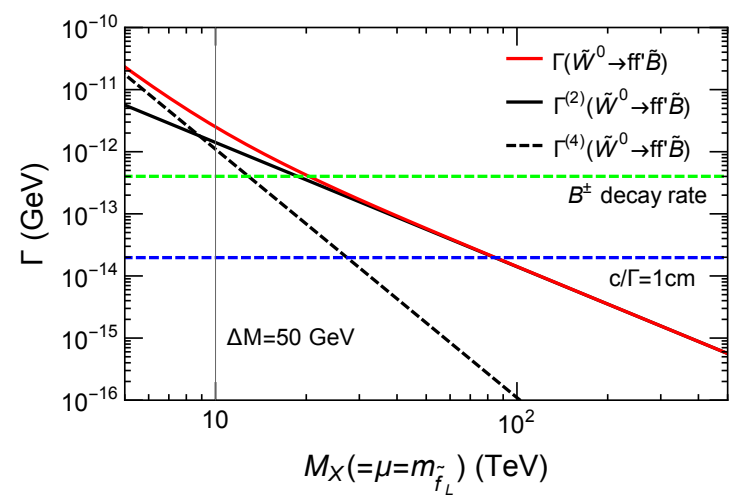

(a)

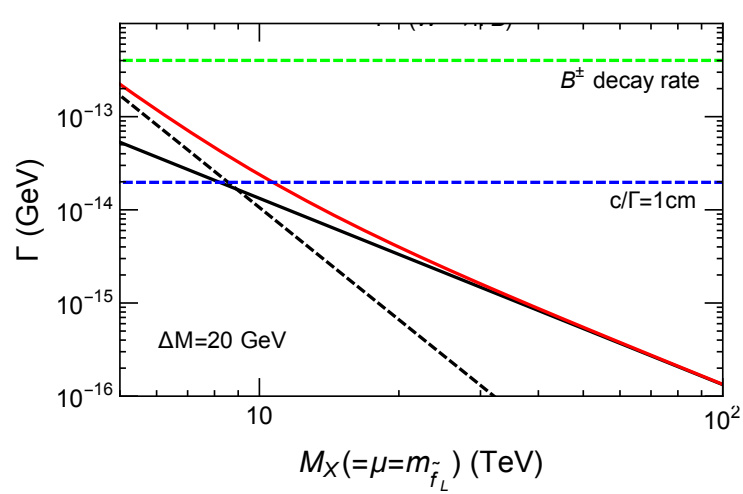

(b)

Figure 7. The three-body decay rate of the neutral wino as a function of $M_{X}\left(=|\mu|=m_{\tilde{f}_{L}}\right)$ for two values of $\Delta M=50 \mathrm{GeV}$ and $20 \mathrm{GeV}$, left and right panel, respectively. The other parameters are taken at $M_{2}=M_{1}+\Delta M, M_{1}=500 \mathrm{GeV}, \tan \beta=2$.

$$
\begin{aligned}
& \hat{\Gamma}_{f}^{V \tilde{f}}\left(\tilde{W}^{0} \rightarrow f \bar{f} \tilde{B}\right) \stackrel{\mu, m_{\tilde{f}_{L}} \gg m_{Z}}{\simeq} \frac{\alpha^{2} M_{2}}{32 \pi} \frac{\hat{\kappa}_{f} \hat{v}_{f} c_{2 \beta}}{s_{2 W}} \frac{M_{2}^{2}}{m_{\tilde{f}_{L}}^{2}} \frac{m_{Z}^{2}}{\mu^{2}} \Omega_{V f}\left(\mu_{\tilde{B}}, \mu_{Z}\right) \\
& \Delta M \lll M_{2}-\frac{\alpha^{2}}{15 \pi} \hat{\kappa}_{f} \hat{v}_{f} \frac{c_{2 \beta}}{s_{2 W}} \frac{(\Delta M)^{5}}{m_{\tilde{f}_{L}}^{2} \mu^{2}},
\end{aligned}
$$

with $\mu_{Z}=m_{Z}^{2} / M_{2}^{2}, \hat{\kappa}_{f}=\left|\hat{e}_{L 1}^{f}\right|\left|\hat{e}_{L 2}^{f}\right|$ and $\hat{v}_{f}=4\left(I_{3}^{f}-e_{f} s_{W}^{2}\right)$, where $I_{3}^{f}$ and $e_{f}$ are the isospin and the electric charge of the sfermion $\tilde{f}$. The $e_{L i}^{f}$ represents the coupling between the left-handed sfermion and the neutralino $\tilde{\chi}_{i}^{0}$, which can be written as

$$
\left(\begin{array}{c}
\hat{e}_{L 1}^{f} \\
\hat{e}_{L 2}^{f}
\end{array}\right)=\sqrt{2}\left[e_{f}\left(\begin{array}{c}
c_{W} \\
s_{W}
\end{array}\right)+\frac{I_{3}^{f}-e_{f} s_{W}^{2}}{c_{W} s_{W}}\left(\begin{array}{c}
-s_{W} \\
c_{W}
\end{array}\right)\right] .
$$

The $\kappa_{Z}^{f}$ is given by $\left(2 I_{3}^{f}-4 e_{f} s_{W}^{2}\right)^{2}+\left(2 I_{3}^{f}\right)^{2}$, namely for each fermion we have

$$
\begin{array}{ll}
u: & \left(1-\frac{8}{3} s_{W}^{2}\right)^{2}+1 \\
d: & \left(-1+\frac{4}{3} s_{W}^{2}\right)^{2}+1 \\
\nu: & 2 \\
e: & \left(-1+4 s_{W}^{2}\right)^{2}+1 .
\end{array}
$$

Analytic forms of $\Omega_{+}, \Omega_{f}$ and $\Omega_{V f}$ are given in appendix A.

The contributions $\Gamma^{(2)}\left(\tilde{W}^{0} \rightarrow f \bar{f} \tilde{B}\right)$ and $\Gamma^{(4)}\left(\tilde{W}^{0} \rightarrow f \bar{f} \tilde{B}\right)$ as well as the total decay rate $\Gamma\left(\tilde{W}^{0} \rightarrow f \bar{f} \tilde{B}\right)$ are shown in figure $7(\mathrm{a})$ and $7(\mathrm{~b})$ for $\Delta M=50$ and $20 \mathrm{GeV}$, respectively, as functions of $M_{X}$, assuming $\mu=m_{\tilde{f}_{L}} \equiv M_{X}$. One can see that the neutral wino can become long lived with $M_{X} \gtrsim 20 \mathrm{TeV}$ for the $\Delta M=50 \mathrm{GeV}$ case and even for $M_{X} \gtrsim 5 \mathrm{TeV}$ for the $\Delta M=20 \mathrm{GeV}$ case. The contribution from the $Z$ and sfermion exchange diagrams, $\Gamma^{(4)}$, is negligible for very large $\mu$. As $M_{X}$ is decreasing, this contribution 


\begin{tabular}{|lcccc|}
\hline & $u$ & $d$ & $e$ & $\nu$ \\
\hline$\hat{\kappa}_{f}^{2}$ & 0.16 & 0.16 & 1.44 & 1.44 \\
$\hat{\kappa}_{f} \hat{v}_{f}$ & 0.56 & 0.68 & -1.33 & -2.40 \\
$\kappa_{Z}^{f}$ & 1.16 & 1.49 & 1.01 & 2 \\
\hline
\end{tabular}

Table 1. Approximate values of coefficients $\hat{\kappa}_{f}^{2}, \hat{\kappa}_{f} \hat{v}_{f}$ and $\kappa_{Z}^{f}$ appearing in eqs. (4.5)-(4.7).

starts to be significant around $M_{X} \sim 20 \mathrm{TeV}$ and becomes the leading contribution from $M_{X} \lesssim 8 \mathrm{TeV}$.

In the limit of $\mu, m_{\tilde{f}_{L}}, m_{A} \gg m_{Z}$ and $\Delta M \ll M_{2}$, the lifetime of the neutral wino can be written approximately as

$$
\begin{array}{ccc}
c \tau_{\tilde{W}^{0}} & \simeq \quad 1 \mathrm{~cm} \cdot\left(\frac{\mu}{10^{2} \mathrm{TeV}}\right)^{2}\left(\frac{50 \mathrm{GeV}}{\Delta M}\right)^{5} S^{-1} \\
\mu \simeq m_{\tilde{f}_{L}}>20 / \sin (2 \beta) \mathrm{TeV} & 1 \mathrm{~cm} \cdot\left(\frac{\mu}{10^{2} \mathrm{TeV}}\right)^{2}\left(\frac{50 \mathrm{GeV}}{\Delta M}\right)^{5}\left(\frac{1}{s_{2 \beta}}\right)^{2},
\end{array}
$$

with

$$
S=s_{2 \beta}^{2}+c_{2 \beta}^{2}\left(\frac{7.87 \mathrm{TeV}}{\mu}\right)^{2}+\sum_{i}\left[\hat{\kappa}_{f_{i}}^{2}\left(\frac{\mu}{m_{\tilde{f}_{L_{i}}}}\right)^{2}\left(\frac{1.56 \mathrm{TeV}}{m_{\tilde{f}_{L_{i}}}}\right)^{2}-c_{2 \beta} \hat{\kappa}_{f_{i}} \hat{v}_{f_{i}}\left(\frac{1.53 \mathrm{TeV}}{m_{\tilde{f}_{L_{i}}}}\right)^{2}\right] .
$$

For $|\mu| \simeq m_{\tilde{f}_{L}}>20 / \sin (2 \beta) \mathrm{TeV}$, the total decay rate is dominated ${ }^{5}$ by $\Gamma^{(2)}$ and in this regime, the lifetime of the neutral wino can be approximated by eq. (4.11). Note that $\hat{\kappa}_{f_{i}}^{2}$ can be $\mathcal{O}(1)$, table 1 , and in case when there is an additional large hierarchy between $\mu$ and $m_{\tilde{f}_{L}}$ the third term of eq. (4.12) might again become relevant.

\subsection{Bino NLSP case $\left(\left|M_{1}\right|>\left|M_{2}\right|\right)$}

In this subsection we assume bino is heavier than winos and define $\Delta M \equiv\left|M_{1}\right|-\left|M_{2}\right|>0$. If $\Delta M<m_{W}$, only three-body bino decays are allowed. As discussed in the previous subsection, the Higgs exchange diagram is suppressed by the mass of the final state fermions, and the $W$-exchange diagram for $\tilde{B} \rightarrow f \bar{f}^{\prime} \tilde{W}^{ \pm}$dominates bino decay. The partial decay rate can be written as

$$
\begin{aligned}
\Gamma_{f}\left(\tilde{B} \rightarrow f \bar{f}^{\prime} \tilde{W}^{ \pm}\right) & =\frac{\alpha^{2} M_{1}}{16 \pi} \frac{1}{s_{W}^{2}} \cdot G_{\tilde{B} \tilde{W}^{ \pm} W}^{2} \cdot \Omega_{-}\left(\mu_{\tilde{W}}, \mu_{W}\right) \\
\mu & \stackrel{\simeq}{\simeq} \frac{\alpha^{2} M_{1}}{16 \pi} \frac{s_{2 \beta}^{2}}{s_{W}^{2}} \frac{m_{Z}^{2}}{\mu^{2}} \frac{m_{W}^{2} \Omega_{-}\left(\mu_{\tilde{W}}, \mu_{W}\right)}{\left(M_{1}-M_{2}\right)^{2}} \\
\Delta M & \geqq M_{1} \frac{4 \alpha^{2}}{15 \pi} \frac{(\Delta M)^{3}}{\mu^{2}} \frac{s_{2 \beta}^{2}}{s_{2 W}^{2}},
\end{aligned}
$$

where $\mu_{W} \equiv m_{W}^{2} / M_{1}^{2}$ and we approximate the expression assuming $\Delta M \ll M_{1}$ on top of $\mu \gg m_{Z}$ in the final step. An analytic formula for $\Omega_{-}\left(\mu_{\tilde{W}}, \mu_{W}\right)$ is provided in appendix A, eqs. (A.5)-(A.9). 


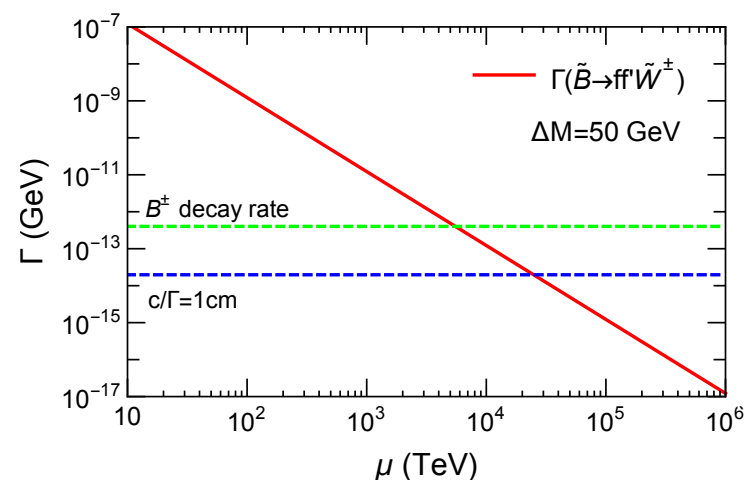

(a)

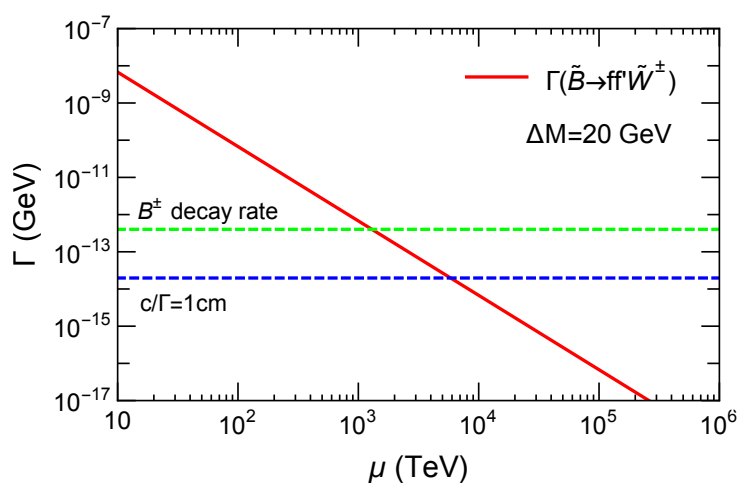

(b)

Figure 8. The three-body decay rate of the bino as a function of $|\mu|$ for two values of $\Delta M=50 \mathrm{GeV}$ and $20 \mathrm{GeV}$, left and right panel, respectively. The other parameters are taken at $M_{1}=M_{2}+\Delta M$, $M_{2}=500 \mathrm{GeV}, \tan \beta=2$.

In figures $8(\mathrm{a})$ and $8(\mathrm{~b})$ we show the bino decay rate $\Gamma\left(\tilde{B} \rightarrow f f^{\prime} \tilde{W}^{ \pm}\right)=\sum_{f} \Gamma_{f}(\tilde{B} \rightarrow$ $\left.f f^{\prime} \tilde{W}^{ \pm}\right)$as a function of $|\mu|$ for $\Delta M=50$ and $20 \mathrm{GeV}$, respectively. As can be seen, the bino becomes long lived for $|\mu|>\mathcal{O}\left(10^{3-4}\right) \mathrm{TeV}$ depending on $\Delta M$. The approximate formula for the bino lifetime is given by

$$
c \tau_{\tilde{B}} \simeq 0.5 \mathrm{~cm} \cdot\left(\frac{\mu}{10^{4} \mathrm{TeV}}\right)^{2}\left(\frac{30 \mathrm{GeV}}{\Delta M}\right)^{3}\left(\frac{1}{s_{2 \beta}}\right)^{2} .
$$

Note that if the decay is mainly mediated by the Higgs boson exchange, the final state will be dominated by (possibly displaced) $b$ or $\tau$ jets.

\section{$5 \quad$ Large mass splitting between gauginos and higgsinos}

In the previous sections, we found that wino and bino can be long-lived for $|\mu| \gtrsim$ $\mathcal{O}\left(10^{5-6}\right) \mathrm{TeV}$ when two-body decays are allowed, and $|\mu| \gtrsim \mathcal{O}\left(10^{1-4}\right) \mathrm{TeV}$ for three-body decays. On the other hand, the wino and bino masses should be less than $\mathcal{O}(1) \mathrm{TeV}$ to be produced at colliders. The long-lived wino and bino in this scenario can be observed only if an enormous mass splitting between gauginos and higgsinos is realised.

The higgsino mass, $\mu$, is the only dimensionfull parameter in the MSSM Lagrangian. Since it is a supersymmetric mass term, its origin can be different from the soft masses of gauginos and scalars. If one does not ask about the origin of the $\mu$ term and does accept the fine tuning in the electroweak symmetry breaking, phenomenologically there is no problem in taking $\mu$ at any value, as long as phenomenologically correct EWSB is achieved.

If $\mu$ is very large, the low energy effective lagrangian is obtained by integrating out the heavy higgsino fields. In doing so, the electroweak gauginos receive the threshold correction from the heavy higgsinos as $[38,39]$

$$
\Delta M_{1}^{\tilde{h}}=\frac{\alpha_{1}}{4 \pi} \frac{3}{5} L, \quad \Delta M_{2}^{\tilde{h}}=\frac{\alpha_{2}}{4 \pi} L,
$$

\footnotetext{
${ }^{5}$ At $|\mu|=m_{\tilde{f}_{L}}=20 \mathrm{TeV}$ and for $\tan \beta=2$ we have $\Gamma^{(4)} / \Gamma^{(2)}=0.195$.
} 
with

$$
L \equiv \frac{\mu m_{A}^{2} s_{2 \beta}}{|\mu|^{2}-m_{A}^{2}} \ln \left(\frac{\left|\mu^{2}\right|}{m_{A}^{2}}\right)
$$

where $m_{A}$ is the CP-odd Higgs mass. If $m_{A}$ and $\mu$ are of the same order and $|\mu| \gtrsim$ $\mathcal{O}\left(10^{3}\right) \mathrm{TeV}$, these threshold corrections exceed $\mathcal{O}(10) \mathrm{TeV}$ and the Wino and Bino become out of the collider reach. In order for these corrections to be small and realise the large mass splitting between heavy higgsinos and light gauginos, one needs $m_{A} \ll|\mu|$. Since $m_{A}$ is related to $\mu$ by

$$
m_{A}^{2}=2|\mu|^{2}+m_{H_{u}}^{2}+m_{H_{d}}^{2},
$$

some unnatural cancellation may be required to accommodate observable long-lived winos and binos for $|\mu| \gtrsim \mathcal{O}\left(10^{3}\right) \mathrm{TeV}$.

Another possibility is to attribute the origin of $\mu$ to the SUSY breaking. For example, in gravity mediation, $\mu$ can be generated as $[40,41]$

$$
\mu=c m_{3 / 2}
$$

where $c$ is a dimensionless coefficient. In this scenario the gaugino masses receive the anomaly mediated contribution [38, 39],

$$
\Delta M_{1}^{\mathrm{AM}}=\frac{\alpha_{1}}{4 \pi} \frac{33}{5} m_{3 / 2}, \quad \Delta M_{2}^{\mathrm{AM}}=\frac{\alpha_{1}}{4 \pi} m_{3 / 2}, \quad \Delta M_{3}^{\mathrm{AM}}=-\frac{\alpha_{1}}{4 \pi} 3 m_{3 / 2},
$$

as well as the higgsino threshold correction of eq. (5.1). The size of these correction exceeds $\mathcal{O}(10) \mathrm{TeV}$ for $|\mu| \gtrsim \mathcal{O}\left(10^{3}\right) \mathrm{TeV}$. In this scenario, some unnatural cancellation among these contributions may be required for the long-lived bino and winos within the collider reach for $|\mu| \gtrsim \mathcal{O}\left(10^{3}\right) \mathrm{TeV}$.

From these considerations one may find the case of the wino NLSP with $\left|M_{2}\right|-\left|M_{1}\right|<$ $m_{Z}$ particularly interesting because the neutral wino can become long-lived for $|\mu| \gtrsim$ $\mathcal{O}\left(10^{1-2}\right) \mathrm{TeV}^{6}$ With $|\mu| \sim \mathcal{O}\left(10^{1-2}\right) \mathrm{TeV}$, the contributions from the higgsino threshold corrections and the anomaly mediation are of the order of $\mathcal{O}\left(10^{2-3}\right) \mathrm{GeV}$ and a large variety of the wino and bino spectra, including compressed spectrum for the three-body decays, can be naturally achieved by the interplay between these contributions [7, 43-45]. In order to precisely predict the lifetime of the neutral wino in this regime, the calculation of the $Z$-exchange diagram induced by the dimension- 5 operator may be necessary. We leave this task for future work.

\section{Collider signatures}

In supersymmetry with heavy scalars and higgsinos, the bino cross section is largely suppressed both at hadron and $e^{+} e^{-}$colliders. The binos may nevertheless be produced from decays of gluinos at hadron colliders. As shown in sections 3 and 4, the lifetime of bino can become larger than $\mathcal{O}(1) \mathrm{cm}$ for $|\mu| \gtrsim \mathcal{O}\left(10^{6}\right) \mathrm{TeV}$ if the two-body decay is allowed and $|\mu| \gtrsim \mathcal{O}\left(10^{4}\right) \mathrm{TeV}$ otherwise.

\footnotetext{
${ }^{6}$ This mass spectrum of bino and wino is also motivated by a recent scan of the 10-dimensional parameter space of phenomenological MSSM [42].
} 
If squarks are heavy the gluinos may also become long-lived. The gluino lifetime is given by [8]:

$$
c \tau_{\tilde{g}}=\mathcal{O}(1 \mathrm{~cm}) \cdot\left(\frac{1 \mathrm{TeV}}{m_{\tilde{g}}}\right)^{5}\left(\frac{m_{\tilde{q}}}{10^{3} \mathrm{TeV}}\right)^{4}
$$

Therefore, if the squark masses and $|\mu|$ are of similar order, the long-lived binos are produced from long-lived gluinos. In order to have the gluino decay well inside the detector, $|\mu|$ and the squark mass cannot be too large. A number of searches have been performed by the LHC experiments looking for metastable gluinos that hadronizes into a colorless $R$ hadron. These include cases when the $R$-hadron escapes a detector before decaying [46, 47], the $R$-hadron is stopped in a detector [26, 48, 49], or it decays inside the detector [50].

Bino can become long-lived already with $|\mu| \sim \mathcal{O}\left(10^{4}\right) \mathrm{TeV}$. In this case the bino predominantly decays into an off-shell $W$ and a charged wino. The charged wino subsequently decays into an off-shell $W$ and the neutral wino, with potentially long lifetime [51, 52]. The charged wino lifetime in this region is given by

$$
c \tau_{\tilde{W}^{ \pm}} \simeq 5 \mathrm{~cm} \cdot\left(\frac{160 \mathrm{MeV}}{\Delta m_{\tilde{W}}}\right)^{3}\left(1-\frac{m_{\pi}^{2}}{\Delta m_{\tilde{W}}^{2}}\right)^{-1 / 2},
$$

where the mass splitting $\Delta m_{\tilde{W}} \equiv m_{\tilde{W}^{ \pm}}-m_{\tilde{W}^{0}}$ can be written as

$$
\Delta m_{\tilde{W}}^{\text {tree }} \sim \frac{1}{4} \operatorname{sign}\left(M_{1} M_{2}\right) \frac{m_{Z}^{4} s_{2 W}^{2} s_{2 \beta}^{2}}{\mu^{2}\left|M_{2}-M_{1}\right|},
$$

at tree-level and receives a radiative correction, $\Delta m_{\tilde{W}}^{\mathrm{rad}} \sim 160 \mathrm{MeV}$ [53]. The long-lived charged winos are searched for by looking for the disappearing track signature $[54,55]$ or measurements of ionisation energy loss in a pixel detector [49]. If the long-lived binos carry the charged winos in the middle of the tracking system $(50-100 \mathrm{~mm})$, the signal can be seen as an appearing-and-disappearing track signature in the detector.

The long-lived wino can be produced either directly at hadron and $e^{+} e^{-}$colliders or indirectly from prompt/non-prompt decays of gluinos at hadron colliders. The charged wino predominantly decays into an on- or off-shell $W$ and the LSP bino. If the decay products of $W^{ \pm(*)}$ are reconstructed, the signal may be detected as a kinked tracks in the leptonic $W^{(*)}$ channel or a displaced dijet with a charged track pointing to the secondary vertex in the hadronic $W^{(*)}$ channel.

The neutral wino predominantly decays into a $h^{(*)}$ and a bino in most cases. The signal should be detected as the displaced jets/dijet signature $[36,56,57]$. It is worth noting that the Higgs mediation can be pinned down by confirming that the displaced jets originate from $b$-quarks and $\tau$-leptons [58-61], as opposed to the $Z$ mediation case, where light jets would dominate the final state. In the region where the neutral wino is long-lived with $|\mu| \sim \mathcal{O}\left(10^{1-2}\right) \mathrm{TeV}$, the two-body decay is kinematically forbidden and the $\tilde{W}^{0}$ decays into a bino and an off-shell $Z, h$ or left-handed sfermions with a small bino-wino mass splitting. Because the decay products in these off-shell decays are soft, the $e^{+} e^{-}$may offer the best opportunity to detect the long-lived neutral wino in this parameter region. At hadron colliders, the search for the displaced $Z^{(*)} \rightarrow \ell \ell$ may also be promising [57, 62, 63]. 


\section{Conclusions}

We investigated a possibility of having long-lived binos and winos in SUSY models with heavy scalars and higgsinos. In the parameter region of interest the $\mathrm{SU}(2)_{L}$ and $\mathrm{U}(1)_{Y}$ gaugino sectors are decoupled from each other with very small mixings proportional to $v / \mu$. In this region, the heavier of bino and wino practically does not interact with the lighter one and its lifetime becomes relevant for collider experiments, $c \tau \gtrsim \mathcal{O}(1) \mathrm{cm}$.

We revisited the decays of bino and winos and found simple formulae for the decay rates and lifetimes, which are valid when the scalars and the higgsinos are much heavier than the gauginos. We have found that the long-lived bino and wino emerge when $|\mu| \gtrsim$ $\mathcal{O}\left(10^{5-6}\right) \mathrm{TeV}$ if the two-body decay mode is open and $|\mu| \gtrsim \mathcal{O}\left(10^{3-4}\right) \mathrm{TeV}$ otherwise. One exception is the case with $0<\left|M_{2}\right|-\left|M_{1}\right|<m_{Z}$, where the lifetime of the neutral wino becomes $\mathcal{O}(1) \mathrm{cm}$ for $|\mu| \sim \mathcal{O}\left(10^{1-2}\right) \mathrm{TeV}$, depending on the wino-bino mass splitting, because the Higgs exchange diagram is suppressed by the mass of the final state fermions. We compared our results to SDecay finding a good agreement for the range of parameters permitted by SDecay.

We briefly discussed how the large mass splitting between gauginos and higgsinos can be achieved. If the origin of $\mu$ is independent of the SUSY breaking, the large mass splitting can be realised relatively easily, although the threshold correction from the heavy higgsinos to the gaugino masses needs to be suppressed. On the other hand, if the $\mu$ is linked to the SUSY breaking and in particular $|\mu| \sim m_{3 / 2}$, the contributions from the higgsino threshold correction and the anomaly mediation become significant. However the large splitting is still possible if one arranges the cancellation between these contributions.

We also discussed a possible collider signature for the long-lived bino and wino in this scenario. The production of bino is only possible from the decay of gluinos, although gluinos tend also to be long-lived for $m_{\tilde{q}} \gtrsim \mathcal{O}\left(10^{3}\right) \mathrm{TeV}$. For the long-lived bino NLSP case, the charged wino may also be long-lived, because of the very small mass splitting within the wino multiplet. In $\tilde{B} \rightarrow W^{ \pm(*)} \tilde{W}^{\mp}$ decay, the bino may carry the long-lived charged winos into the middle of the tracking system and the signal could be seen as an appearing-and-disappearing track signature.

For the wino NLSP case, the production of winos is possible either directly or indirectly from the gluino decay. The long-lived charged wino decaying to $W^{ \pm(*)}$ and $\tilde{B}$ can be detected as a kinked-track signature from the leptonic decay of $W^{(*)}$. The hadronic $W^{(*)}$ mode may also be seen as events with a displaced dijet and a track pointing to the secondary vertex. Detecting the long-lived neutral wino with $|\mu| \sim \mathcal{O}\left(10^{1-2}\right) \mathrm{TeV}$ may be challenging because the decay products will be soft due to the small wino-bino mass splitting. In this case, $e^{+} e^{-}$collider may be ideal to detect the long-lived neutral wino. Otherwise a displaced off-shell $Z^{*} \rightarrow \ell \ell$ decay may be promising even at hadron colliders. 


\section{Acknowledgments}

K.S. thanks Satoshi Shirai for valuable discussion. K.S. is supported in part by the London Centre for Terauniverse Studies (LCTS), using funding from the European Research Council via the Advanced Investigator Grant 267352. K.R. has been supported by the MINECO (Spain) under contract FPA2013-44773-P; Consolider-Ingenio CPAN CSD200700042; the Spanish MINECO Centro de excelencia Severo Ochoa Program under grant SEV-2012-0249; and by JAE-Doc program.

\section{A Auxiliary functions}

In this appendix we summarize the analytic expressions for functions used in the calculation of decay widths.

$$
\begin{aligned}
& f_{ \pm}(x, y)=\sqrt{\lambda(x, y)} \eta_{ \pm}(x, y) \\
& \lambda(x, y)=1+x^{2}+y^{2}-2 x-2 y-2 x y, \\
& \eta_{ \pm}(x, y)=(1+x-y)+\frac{(1-x+y)(1-x-y)}{y} \pm 6 \sqrt{x}, \\
& f_{h}(x, y)=(1+x-y+2 \sqrt{x}) \sqrt{\lambda(x, y)}, \\
& \Omega_{ \pm}(x, y)=2 F(x, y) \pm G(x, y) \text {, } \\
& F(x, y)=\frac{x-1}{6 y}[\omega(x, y)+y(5+5 x-7 y)] \\
& -\frac{y}{2}\{(1+x-y) \ln x+[\omega(x, y)+2 x] \mathcal{L}(x, y)\}, \\
& G(x, y)=\sqrt{x}[4(x-1)+(1+x-2 y) \ln x+\{\omega(x, y)-y(1+x-y)\} \mathcal{L}(x, y)], \\
& \omega(x, y)=1-2 x-2 y+(y-x)^{2}, \\
& \mathcal{L}(x, y)=\frac{2}{\sqrt{-\omega(x, y)}}\left[\arctan \left(\frac{-1+x-y}{\sqrt{-\omega(x, y)}}\right)-\arctan \left(\frac{1-x-y}{\sqrt{-\omega(x, y)}}\right)\right], \\
& \Omega_{h}(x, y)=H(x, y)+G(x, y) \text {, } \\
& H(x, y)=\frac{1}{2}(1-x)(6 y-5-5 x)+\frac{1}{2}\left(1-4 y-4 x y+3 y^{2}+x^{2}\right) \ln x \\
& +\frac{1}{2}\left(-5 x^{2} y-3 y^{3}+7 y^{2}+1-x^{2}-x+x^{3}-5 y+7 x y^{2}-2 x y\right) \mathcal{L}(x, y), \\
& \Omega_{f}(x)=\frac{1}{6}\left(1-8 x+8 x^{3}-x^{4}-12 x^{2} \ln x\right) \\
& +\frac{\sqrt{x}}{6}\left[1+x\left(9-9 x-x^{2}\right)+6 x(1+x) \ln x\right], \\
& \Omega_{V}(x)=\frac{1}{36}\left(-5-9 x+9 x^{2}+5 x^{3}+\left(3-9 x-18 x^{2}\right) \ln x\right) \\
& -\frac{(x-1) \sqrt{4 x-1}}{6}\left[\arctan \left(\frac{1}{\sqrt{4 x-1}}\right)+\arctan \left(\frac{1-2 x}{\sqrt{4 x-1}}\right)\right],
\end{aligned}
$$




$$
\begin{aligned}
& \Omega_{V f}(x, y)=(1+\sqrt{x})[ {\left[1-3 x-3 x^{2}+x^{3}-3(1+x) y^{2}+2 y^{3}\right] \frac{\ln x}{12} } \\
&+\left[5+5 x^{2}-12(y-1) y+2 x(1+6 y)\right] \frac{x-1}{36} \\
&+\left[1+x^{2}+x(y-2)+y-2 y^{2}\right]\left[x^{2}+(y-1)^{2}-2 x(1+y)\right] \\
&\left.\times \frac{\mathcal{M}(x, y)}{6 \sqrt{-x^{2}-(y-1)^{2}+2 x(1+y)}}\right] \\
& \mathcal{M}(x, y)=\arctan \left[\frac{-1+x+y}{\sqrt{-x^{2}-(y-1)^{2}+2 x(1+y)}}\right] \\
&-\arctan \left[\frac{1-x+y}{\sqrt{-x^{2}-(y-1)^{2}+2 x(1+y)}}\right]
\end{aligned}
$$

\section{B Comparison with SDecay}

In this section we compare approximated results obtained in this paper with exact calculation performed using the computer code SDecay [27]. Figure 9 shows the partial decay widths using both approaches for processes: $\tilde{W}^{ \pm} \rightarrow W^{ \pm} \tilde{B}$ (cf. section 3.1), $\tilde{W}^{+} \rightarrow e^{+} \nu \tilde{B}$ (cf. section 4.1), and $\tilde{W}^{0} \rightarrow b \bar{b} \tilde{B}$ (cf. section 4.1). For the last process we remove the squark mediated contribution by taking $m_{\tilde{f}_{L}}=10|\mu|$ and approximate $\Gamma=N_{C}\left(\hat{\Gamma}_{b}^{h}+\hat{\Gamma}_{b}^{Z}\right)$, where $N_{C}(=3)$ is the colour factor. We use eqs. (4.4) and (4.5) for $\hat{\Gamma}_{b}^{h}$ and $\hat{\Gamma}_{b}^{Z}$, respectively. In addition, we show the ratio of decay widths,

$$
R=\frac{\Gamma^{\text {approx }}}{\Gamma^{\text {SDecay }}}
$$

in the lower panels of each plot. In order to ensure that the spectra and couplings are the same for the purpose of comparison, we calculate the spectrum fed to SDecay externally to avoid corrections due to running parameters. For sufficiently large values of $\mu$ we find a very good agreement between both predictions, within $\mathcal{O}(10 \%)$. A level of $\mathcal{O}(40 \%)$ agreement can be also achieved for lower values of $\mu$. An important thing to note is that the calculation by SDecay fails for $\mu \gtrsim 10^{5} \mathrm{TeV}$, due to numerical problems. Another point to note is that because of the large separation between gaugino and stops scales one would typically run into problems with standard spectrum calculators. For these reasons using approximate formulae would be preferred over automated codes in the large $\mu$ regime. 


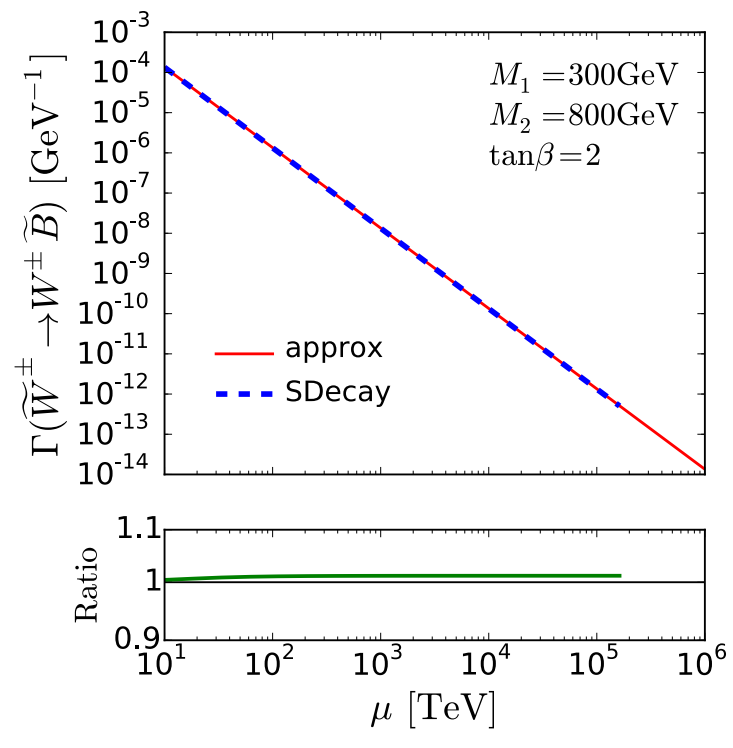

(a)

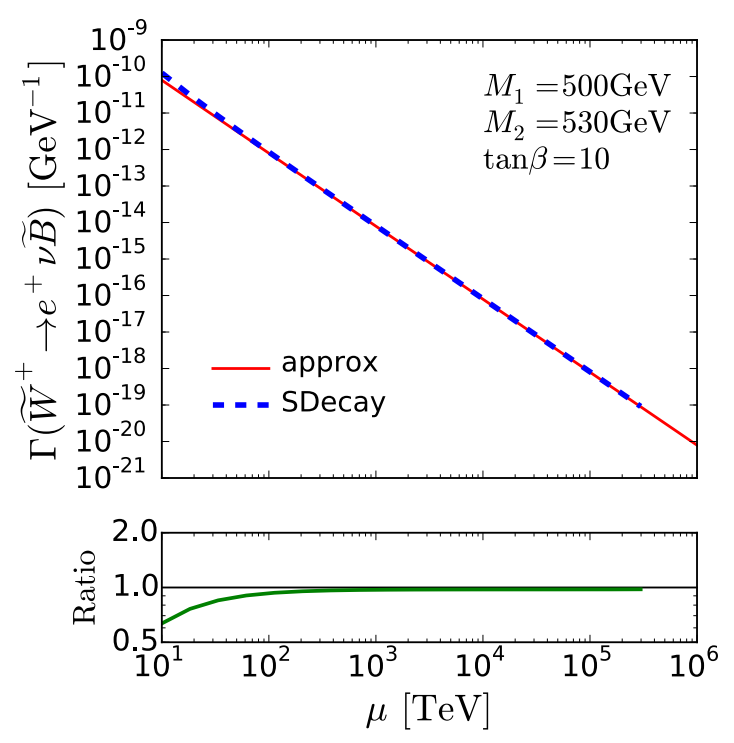

(b)

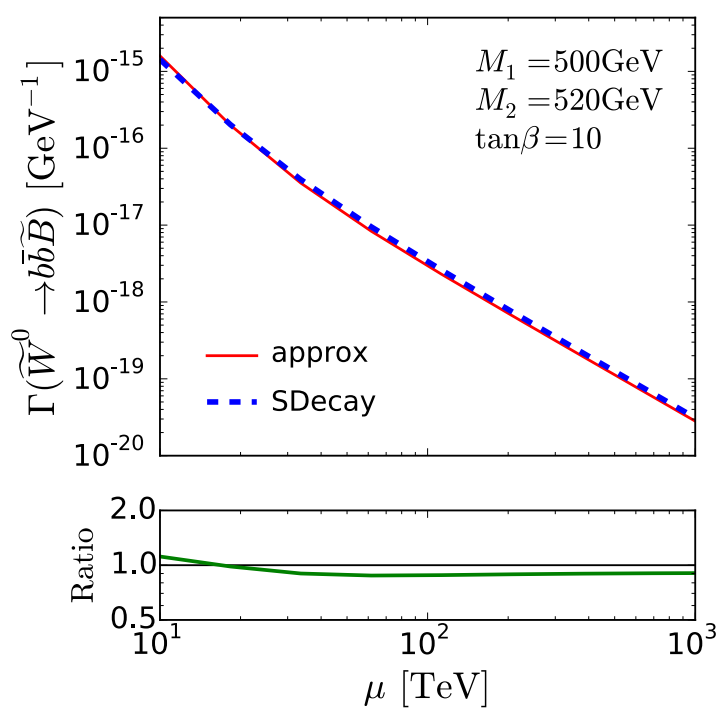

(c)

Figure 9. Comparison of partial decay widths obtained using approximated formulae (red solid line) and SDecay [27] (blue dashed line) for different decay channels. In the lower panel of each plot the ratio of respective decay widths is shown, as defined in eq. (B.1). 
Open Access. This article is distributed under the terms of the Creative Commons Attribution License (CC-BY 4.0), which permits any use, distribution and reproduction in any medium, provided the original author(s) and source are credited.

\section{References}

[1] ATLAS collaboration, Observation of a new particle in the search for the standard model Higgs boson with the ATLAS detector at the LHC, Phys. Lett. B 716 (2012) 1 [arXiv: 1207.7214] [INSPIRE].

[2] CMS collaboration, Observation of a new boson at a mass of $125 \mathrm{GeV}$ with the CMS experiment at the LHC, Phys. Lett. B 716 (2012) 30 [arXiv:1207.7235] [INSPIRE].

[3] CMS collaboration, Precise determination of the mass of the Higgs boson and tests of compatibility of its couplings with the standard model predictions using proton collisions at 7 and $8 \mathrm{TeV}$, Eur. Phys. J. C 75 (2015) 212 [arXiv:1412.8662] [inSPIRE].

[4] ATLAS and CMS collaborations, Combined measurement of the Higgs boson mass in pp collisions at $\sqrt{s}=7$ and $8 \mathrm{TeV}$ with the ATLAS and CMS experiments, Phys. Rev. Lett. 114 (2015) 191803 [arXiv:1503.07589] [INSPIRE].

[5] G.F. Giudice and A. Strumia, Probing high-scale and split supersymmetry with Higgs mass measurements, Nucl. Phys. B 858 (2012) 63 [arXiv:1108.6077] [InSPIRE].

[6] M. Ibe, S. Matsumoto and T.T. Yanagida, Pure gravity mediation with $m_{3 / 2}=10-100 \mathrm{TeV}$, Phys. Rev. D 85 (2012) 095011 [arXiv:1202.2253] [InSPIRE].

[7] E. Bagnaschi, G.F. Giudice, P. Slavich and A. Strumia, Higgs mass and unnatural supersymmetry, JHEP 09 (2014) 092 [arXiv: 1407.4081] [INSPIRE].

[8] N. Arkani-Hamed and S. Dimopoulos, Supersymmetric unification without low energy supersymmetry and signatures for fine-tuning at the LHC, JHEP 06 (2005) 073 [hep-th/0405159] [INSPIRE].

[9] J. Hisano, S. Matsumoto, M. Nagai, O. Saito and M. Senami, Non-perturbative effect on thermal relic abundance of dark matter, Phys. Lett. B 646 (2007) 34 [hep-ph/0610249] [INSPIRE].

[10] M. Cirelli, A. Strumia and M. Tamburini, Cosmology and astrophysics of minimal dark matter, Nucl. Phys. B 787 (2007) 152 [arXiv:0706.4071] [INSPIRE].

[11] T. Cohen, M. Lisanti, A. Pierce and T.R. Slatyer, Wino dark matter under siege, JCAP 10 (2013) 061 [arXiv: 1307.4082] [INSPIRE].

[12] C. Cheung, L.J. Hall, D. Pinner and J.T. Ruderman, Prospects and blind spots for neutralino dark matter, JHEP 05 (2013) 100 [arXiv:1211.4873] [INSPIRE].

[13] M. Berggren et al., Tackling light higgsinos at the ILC, Eur. Phys. J. C 73 (2013) 2660 [arXiv: 1307.3566] [INSPIRE].

[14] T. Han, S. Padhi and S. Su, Electroweakinos in the light of the Higgs boson, Phys. Rev. D 88 (2013) 115010 [arXiv:1309.5966] [InSPIRE].

[15] P. Schwaller and J. Zurita, Compressed electroweakino spectra at the LHC, JHEP 03 (2014) 060 [arXiv: 1312.7350] [INSPIRE].

[16] M. Low and L.-T. Wang, Neutralino dark matter at $14 \mathrm{TeV}$ and $100 \mathrm{TeV}$, JHEP 08 (2014) 161 [arXiv: 1404.0682] [InSPIRE]. 
[17] A. Anandakrishnan, L.M. Carpenter and S. Raby, Degenerate gaugino mass region and mono-boson collider signatures, Phys. Rev. D 90 (2014) 055004 [arXiv:1407.1833] [INSPIRE].

[18] J. Bramante, A. Delgado, F. Elahi, A. Martin and B. Ostdiek, Catching sparks from well-forged neutralinos, Phys. Rev. D 90 (2014) 095008 [arXiv:1408.6530] [InSPIRE].

[19] T.A.W. Martin and D. Morrissey, Electroweakino constraints from LHC data, JHEP 12 (2014) 168 [arXiv:1409.6322] [INSPIRE].

[20] B.S. Acharya, K. Bożek, C. Pongkitivanichkul and K. Sakurai, Prospects for observing charginos and neutralinos at a 100 TeV proton-proton collider, JHEP 02 (2015) 181 [arXiv: 1410.1532] [INSPIRE].

[21] G. Grilli di Cortona, Hunting electroweakinos at future hadron colliders and direct detection experiments, JHEP 05 (2015) 035 [arXiv: 1412.5952] [INSPIRE].

[22] C. Han, D. Kim, S. Munir and M. Park, Accessing the core of naturalness, nearly degenerate higgsinos, at the LHC, JHEP 04 (2015) 132 [arXiv: 1502.03734] [INSPIRE].

[23] D. Barducci, A. Belyaev, A.K.M. Bharucha, W. Porod and V. Sanz, Uncovering natural supersymmetry via the interplay between the LHC and direct dark matter detection, JHEP 07 (2015) 066 [arXiv: 1504.02472] [INSPIRE].

[24] M. Badziak, A. Delgado, M. Olechowski, S. Pokorski and K. Sakurai, Detecting underabundant neutralinos, arXiv: 1506.07177 [INSPIRE].

[25] J.L. Hewett, B. Lillie, M. Masip and T.G. Rizzo, Signatures of long-lived gluinos in split supersymmetry, JHEP 09 (2004) 070 [hep-ph/0408248] [INSPIRE].

[26] ATLAS collaboration, Search for long-lived stopped R-hadrons decaying out-of-time with $p p$ collisions using the ATLAS detector, Phys. Rev. D 88 (2013) 112003 [arXiv:1310.6584] [INSPIRE].

[27] M. Mühlleitner, A. Djouadi and Y. Mambrini, SDECAY: a Fortran code for the decays of the supersymmetric particles in the MSSM, Comput. Phys. Commun. 168 (2005) 46 [hep-ph/0311167] [INSPIRE].

[28] J.F. Gunion and H.E. Haber, Errata for Higgs bosons in supersymmetric models: I, II and III, hep-ph/9301205 [INSPIRE].

[29] A. Djouadi, Y. Mambrini and M. Mühlleitner, Chargino and neutralino decays revisited, Eur. Phys. J. C 20 (2001) 563 [hep-ph/0104115] [INSPIRE].

[30] H.E. Haber, Challenges for nonminimal Higgs searches at future colliders, hep-ph/9505240 [INSPIRE].

[31] A. Djouadi, J. Kalinowski, P. Ohmann and P.M. Zerwas, Heavy SUSY Higgs bosons at $e^{+} e^{-}$ linear colliders, Z. Phys. C 74 (1997) 93 [hep-ph/9605339] [INSPIRE].

[32] A. Djouadi, The anatomy of electro-weak symmetry breaking. II. The Higgs bosons in the minimal supersymmetric model, Phys. Rept. 459 (2008) 1 [hep-ph/0503173] [INSPIRE].

[33] CMS collaboration, Description and performance of track and primary-vertex reconstruction with the CMS tracker, 2014 JINST 9 P10009 [arXiv:1405.6569] [INSPIRE].

[34] ATLAS collaboration, Alignment of the ATLAS inner detector and its performance in 2012, ATLAS-CONF-2014-047 (2014). 
[35] H. Abramowicz et al., The International Linear Collider technical design report. Volume 4: Detectors, arXiv:1306.6329 [INSPIRE].

[36] CMS collaboration, Search for long-lived neutral particles decaying to quark-antiquark pairs in proton-proton collisions at $\sqrt{s}=8 \mathrm{TeV}$, Phys. Rev. D 91 (2015) 012007 [arXiv: 1411.6530] [INSPIRE].

[37] ATLAS collaboration, Search for pair-produced long-lived neutral particles decaying in the ATLAS hadronic calorimeter in pp collisions at $\sqrt{s}=8$ TeV, Phys. Lett. B 743 (2015) 15 [arXiv: 1501.04020] [INSPIRE].

[38] L. Randall and R. Sundrum, Out of this world supersymmetry breaking, Nucl. Phys. B 557 (1999) 79 [hep-th/9810155] [INSPIRE].

[39] G.F. Giudice, M.A. Luty, H. Murayama and R. Rattazzi, Gaugino mass without singlets, JHEP 12 (1998) 027 [hep-ph/9810442] [INSPIRE].

[40] G.F. Giudice and A. Masiero, A natural solution to the $\mu$-problem in supergravity theories, Phys. Lett. B 206 (1988) 480 [INSPIRE].

[41] K. Inoue, M. Kawasaki, M. Yamaguchi and T. Yanagida, Vanishing squark and slepton masses in a class of supergravity models, Phys. Rev. D 45 (1992) 328 [INSPIRE].

[42] K.J. de Vries et al., The pMSSM10 after LHC Run 1, Eur. Phys. J. C 75 (2015) 422 [arXiv: 1504.03260] [INSPIRE].

[43] B. Bhattacherjee, B. Feldstein, M. Ibe, S. Matsumoto and T.T. Yanagida, Pure gravity mediation of supersymmetry breaking at the Large Hadron Collider, Phys. Rev. D 87 (2013) 015028 [arXiv: 1207.5453] [INSPIRE].

[44] L.J. Hall, Y. Nomura and S. Shirai, Spread supersymmetry with wino LSP: gluino and dark matter signals, JHEP 01 (2013) 036 [arXiv:1210.2395] [INSPIRE].

[45] K. Harigaya, K. Kaneta and S. Matsumoto, Gaugino coannihilations, Phys. Rev. D 89 (2014) 115021 [arXiv:1403.0715] [InSPIRE].

[46] ATLAS collaboration, Searches for heavy long-lived sleptons and R-Hadrons with the ATLAS detector in pp collisions at $\sqrt{s}=7 \mathrm{TeV}$, Phys. Lett. B 720 (2013) 277 [arXiv: 1211.1597] [INSPIRE].

[47] CMS collaboration, Searches for long-lived charged particles in pp collisions at $\sqrt{s}=7$ and 8 TeV, JHEP 07 (2013) 122 [arXiv:1305.0491] [INSPIRE].

[48] CMS collaboration, Search for decays of stopped long-lived particles produced in proton-proton collisions at $\sqrt{s}=8 \mathrm{TeV}$, Eur. Phys. J. C 75 (2015) 151 [arXiv:1501.05603] [INSPIRE].

[49] ATLAS collaboration, Search for metastable heavy charged particles with large ionisation energy loss in pp collisions at $\sqrt{s}=8 \mathrm{TeV}$ using the ATLAS experiment, Eur. Phys. J. C 75 (2015) 407 [arXiv:1506.05332] [inSPIRE].

[50] ATLAS collaboration, Limits on metastable gluinos from ATLAS SUSY searches at $8 \mathrm{TeV}$, ATLAS-CONF-2014-037 (2014).

[51] M. Ibe, T. Moroi and T.T. Yanagida, Possible signals of wino LSP at the Large Hadron Collider, Phys. Lett. B 644 (2007) 355 [hep-ph/0610277] [INSPIRE].

[52] M.R. Buckley, L. Randall and B. Shuve, LHC searches for non-chiral weakly charged multiplets, JHEP 05 (2011) 097 [arXiv: 0909.4549] [INSPIRE]. 
[53] M. Ibe, S. Matsumoto and R. Sato, Mass splitting between charged and neutral winos at two-loop level, Phys. Lett. B 721 (2013) 252 [arXiv:1212.5989] [INSPIRE].

[54] ATLAS collaboration, Search for charginos nearly mass degenerate with the lightest neutralino based on a disappearing-track signature in pp collisions at $\sqrt{s}=8 \mathrm{TeV}$ with the ATLAS detector, Phys. Rev. D 88 (2013) 112006 [arXiv:1310.3675] [INSPIRE].

[55] CMS collaboration, Search for disappearing tracks in proton-proton collisions at $\sqrt{s}=8 \mathrm{TeV}$, JHEP 01 (2015) 096 [arXiv:1411.6006] [INSPIRE].

[56] ATLAS collaboration, Search for long-lived, weakly interacting particles that decay to displaced hadronic jets in proton-proton collisions at $\sqrt{s}=8 \mathrm{TeV}$ with the ATLAS detector, Phys. Rev. D 92 (2015) 012010 [arXiv: 1504.03634] [INSPIRE].

[57] ATLAS collaboration, Search for massive, long-lived particles using multitrack displaced vertices or displaced lepton pairs in pp collisions at $\sqrt{s}=8 \mathrm{TeV}$ with the ATLAS detector, Phys. Rev. D 92 (2015) 072004 [arXiv: 1504.05162] [INSPIRE].

[58] ATLAS collaboration, Calibration of the performance of b-tagging for $c$ and light-flavour jets in the 2012 ATLAS data, ATLAS-CONF-2014-046 (2014).

[59] ATLAS collaboration, Identification and energy calibration of hadronically decaying tau leptons with the ATLAS experiment in pp collisions at $\sqrt{s}=8 \mathrm{TeV}$, Eur. Phys. J. C 75 (2015) 303 [arXiv:1412.7086] [INSPIRE].

[60] CMS collaboration, Identification of b-quark jets with the CMS experiment, 2013 JINST 8 P04013 [arXiv:1211.4462] [INSPIRE].

[61] CMS collaboration, Performance of tau-lepton reconstruction and identification in CMS, 2012 JINST 7 P01001 [arXiv: 1109.6034] [INSPIRE].

[62] CMS collaboration, Search for long-lived particles that decay into final states containing two electrons or two muons in proton-proton collisions at $\sqrt{s}=8 \mathrm{TeV}$, Phys. Rev. D 91 (2015) 052012 [arXiv:1411.6977] [INSPIRE].

[63] CMS collaboration, Search for long-lived particles that decay into final states containing two muons, reconstructed using only the CMS muon chambers, CMS-PAS-EXO-14-012 (2015). 